\title{
Binary Sensing Matrix Design for Compressive Imaging Measurements
}

\author{
Jun Ke ${ }^{* 1}$, Ping Wei ${ }^{\star 1}$ and Edmund Y. Lam ${ }^{\dagger 2}$ \\ ${ }^{1}$ School of Optoelectronics, Beijing Institute of Technology, Beijing, China \\ ${ }^{2}$ Department of Electrical and Electronic Engineering, The University of Hong Kong, Pokfulam, Hong Kong \\ *jke@bit.edu.cn; *pwei@bit.edu.cn; †elam@eee.hku.hk
}

\begin{abstract}
We design a binary sensing matrix in compressive imaging to reduce the capture time while maintaining image reconstruction performance, by minimizing the distance between the binary matrix and a modified principal component analysis sensing matrix.

OCIS codes: (110.1758) Computational imaging, (100.3010) Image reconstruction technique.
\end{abstract}

\section{Introduction}

Sensing matrix design is one important topic in compressive imaging (CI) research. Most sensing matrix design works [1-3] focus on obtaining a better reconstruction. However, in CI, another important issue is the time consuming measurement collecting process. To reduce measurement collecting time, we search a binary sensing matrix for CI. Different from the random binary matrix which has elements selected from a Bernoulli distribution, the matrix we obtained presents a reconstruction mean square error (MSE) close to the optimal value. Therefore the matrix benefits system reconstruction besides fastening measurement collecting process. The paper is organized as following. The binary matrix design work is discussed in section 2 . Then numberical simulation results are presented in section 3 with conclusions drawn in section 4.

\section{Binary sensing matrix design}

In compressive imaging, system measurements can be represented as

$$
\vec{y}=\mathrm{F} \vec{x}+\vec{n},
$$

where $\vec{y}(M \times 1), \vec{x}(N \times 1)$ and $\vec{n}(M \times 1)$ are the measurement, the object, and noise vectors, respectively. $\mathrm{F}(M \times N)$ is the sensing matrix. The noise is assumed as additive white Gaussian noise $\mathscr{N}\left(0, \sigma^{2}\right)$.

To collect feature $\vec{y}$, different kinds of sensing matrices, such as random Gaussian, random binary matrices have been studied. However, the performance of these matrices degrade fast when noise is high. On the other hand, principal component analysis (PCA) sensing matrix has been proved to be the optimal in the sense of minimizing reconstruction error as noise presents. Therefore, we focus on PCA in this work. Note that, since PCA is the optimal for reconstruction, if a sensing matrix is close to PCA, then it will have good reconstruction performance. Thus we define the binary sensing matrix design problem as searching a matrix similar to PCA. In our previous work [4], we also notice that, if $F=Q_{P C A}^{T}$ is the PCA sensing matrix, then the matrix $A_{P C A}^{T}$, where $A$ is invertible, will generate the same system reconstruction MSE. With all these observations, we can solve the following problem for binary matrix design,

$$
\begin{aligned}
\mathbf{P}: \quad \min _{\mathrm{A}} & \varepsilon(\mathrm{A})=\left\|\sqrt{N} \mathrm{AQ}_{\mathrm{PCA}}^{\mathrm{T}}-\operatorname{sgn}\left(\mathrm{AQ}_{\mathrm{PCA}}^{\mathrm{T}}\right)\right\|_{F}^{2} \\
\text { subj to } & \mathrm{AA}^{\mathrm{T}}=\mathrm{I},
\end{aligned}
$$

where $\|\cdot\|_{F}$ represents the Frobenius norm of a matrix, and $\operatorname{sgn}()$ is the signum function. We redefine the constraint as $G=0$, where $G=A A^{T}-I$. Then the Lagrangian for $\mathbf{P}$ can be written as

$$
\mathscr{L}(\mathrm{A}, \lambda)=\sum_{i=1}^{M} \sum_{j=1}^{N}\left\lfloor\sqrt{N} \mathrm{AQ}_{\mathrm{PCA}}^{\mathrm{T}}-\operatorname{sgn}\left\{\mathrm{AQ}_{\mathrm{PCA}}^{\mathrm{T}}\right\}\right\rfloor_{i j}^{2}+\sum_{i=1}^{M} \sum_{j=i}^{M}\lfloor\lambda \cdot * \mathrm{G}\rfloor_{i j},
$$

where $L \cdot\rfloor_{i j}$ represents the $(i, j)_{\text {th }}$ element of a matrix, $\lambda$ is a symmetric matrix of size $(M \times M)$, and $* *$ represents the element multiplication between two matrices.

$\mathbf{P}$ is an equality-constrained optimization problem. To solve $\mathbf{P}$, we use the Newton-Raphson method [5], which can be represented using the following equations,

$$
\left[\begin{array}{cc}
\nabla_{\mathrm{AA}}^{2} \mathscr{L}\left(\vec{a}^{(v)}, \vec{\lambda}^{(v)}\right) & J\left(\vec{a}^{(v)}\right)^{\mathrm{T}} \\
J\left(\vec{a}^{(v)}\right) & 0
\end{array}\right]\left[\begin{array}{c}
\Delta \vec{a}^{(v)} \\
\Delta \vec{\lambda}^{(v)}
\end{array}\right]=-\left[\begin{array}{c}
\nabla \varepsilon\left(\vec{a}^{(v)}\right)+J\left(\vec{a}^{(v)}\right)^{\mathrm{T}} \vec{\lambda}^{(v)} \\
\vec{g}\left(\vec{a}^{(v)}\right)
\end{array}\right],
$$


and

$$
\left[\begin{array}{l}
\vec{a}^{(v+1)} \\
\vec{\lambda}^{(v+1)}
\end{array}\right]=\left[\begin{array}{c}
\Delta \vec{a}^{(v)} \\
\Delta \vec{\lambda}^{(v)}
\end{array}\right]+\alpha\left[\begin{array}{c}
\vec{a}^{(v)} \\
\vec{\lambda}^{(v)}
\end{array}\right] .
$$

In Eq. 4 and 5, $\nabla_{\mathrm{AA}}^{2} \mathscr{L}$ is the Hessian matrix of the Lagrangian, $J$ is the Jacobi of the constraint for $\mathbf{P}_{3}$, and $\nabla \varepsilon$ is the gradient vector of the cost function. Different from Eq. 2, the variables in Eq. 4 and 5 are vectors $\vec{a}$ and $\vec{\lambda}$, which are obtained by lexicographically ordering the elements of $A$ and the upper triangle part of $\lambda$.

The element of the Hessian matrix $\nabla_{\mathrm{AA}}^{2} \mathscr{L}\left(\vec{a}^{(v)}, \vec{\lambda}^{(v)}\right)$ can be written as

$$
\frac{\partial^{2} \mathscr{L}(\vec{a}, \vec{\lambda})}{\partial \mathrm{A}_{i j} \partial \mathrm{A}_{i^{\prime} j^{\prime}}}=2 N \delta\left[i-i^{\prime}\right] \delta\left[j-j^{\prime}\right]+2 \lambda_{i i^{\prime}} \delta\left[j-j^{\prime}\right],
$$

where $\delta[i]$ is the delta function. For the Jacobi matrix of $\mathrm{G}$, note that $\mathrm{G}$ is symmetric. Therefore only the upper triangle part of $\mathrm{G}$ is used to find $J$. We have

$$
\frac{\partial \mathrm{G}_{i j}}{\partial \mathrm{A}_{i^{\prime} j^{\prime}}}=\delta\left[i-i^{\prime}\right] \mathrm{A}_{j j^{\prime}}+\delta\left[j-i^{\prime}\right] \mathrm{A}_{i j^{\prime}}
$$

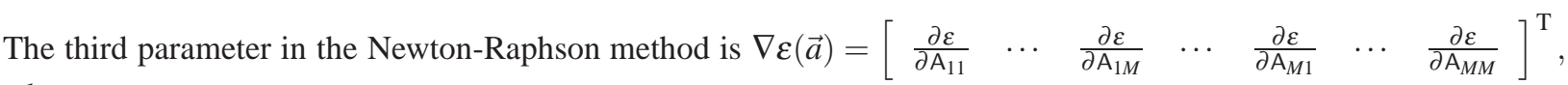
where

$$
\frac{\partial \varepsilon}{\partial \mathrm{A}_{i j}}=2 N \mathrm{~A}_{i j}-2 \sqrt{N}\left\lfloor\operatorname{sgn}\left(\mathrm{AQ}_{\mathrm{PCA}}^{\mathrm{T}}\right) \mathrm{Q}_{\mathrm{PCA}}\right\rfloor_{i j}
$$

\section{Numerical experiment}

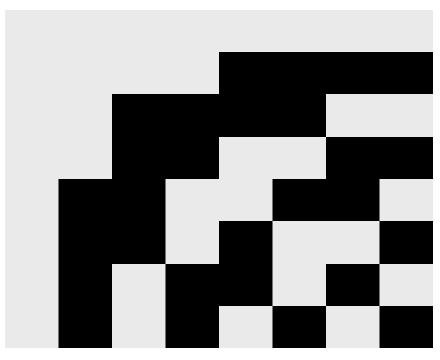

(a)

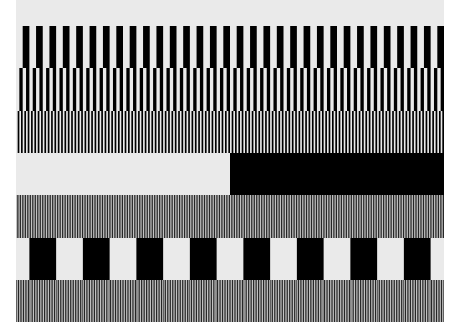

(b)

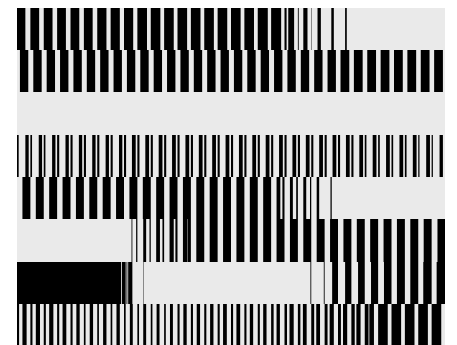

(c)

Fig. 1. (a) The Hadamard vectors sorted based on the variation of each vector, (b) the Hadamard vector sorted by the mean of a training set, (c) the optimal binary vector set or the optimal solution for problem $\mathbf{P}$, when $M$ is $M=8$ for $32 \times 32$ blocks.

We use linear Wiener operator for object reconstruction due to its faster computational speed and equally reconstruction performance as nonlinear methods for high noise case [6]. The Wiener operator is defined as $\mathrm{W}=$ $\mathrm{R}_{\vec{x}} \mathrm{~F}^{\mathrm{T}}\left(\mathrm{FR}_{\vec{x}} \mathrm{~F}^{\mathrm{T}}+\sigma^{2} \mathrm{I}\right)^{-1}$. Using $\mathrm{W}$, the object can be reconstructed as $\vec{x}_{\text {est }}=\mathrm{W} \vec{y}$.

An object of size $(1056 \times 1920)$ is used with block-based low-light-level compressive imaging $(\mathrm{BCL}-\mathrm{Imaging})[7,8]$ for the experiment. The block size is $(32 \times 32) . M=8$ features are searched and used to collect system measurements. The noise level is $\sigma=150$. Fig. 1 presents three binary sensing matrices. Both (a) and (b) are Hadamard vectors. However, the vectors in (a) are sorted using the variation frequency in each vector, while the results in (b) are ordered based on the projections of a block sample average $\mathbf{x}_{\text {mean }}$ over Hadamard vectors. Fig. 1 (c) shows the searching result by solving problem P. Reconstructions using all these binary matrices and PCA are presented in Fig. 2. In Fig. 2 (a), the upper image is the reconstruction using the searched optimal binary sensing matrix, while the lower uses PCA. It can be observed both have similar visual quality. Fig. 2 (b) and (c) show two detail parts of the reconstructions using all four matrices. From top to bottom, the sub-figures correspond to Fig. 1 (a), (b), (c) and PCA, respectively. The error values for the four reconstructions are $0.916,0.753,0.707$, and 0.692 . It is clear, the solution to problem $\mathbf{P}$ shows the best reconstruction among the three binary matrices. Its performance is also very similar to PCA. 

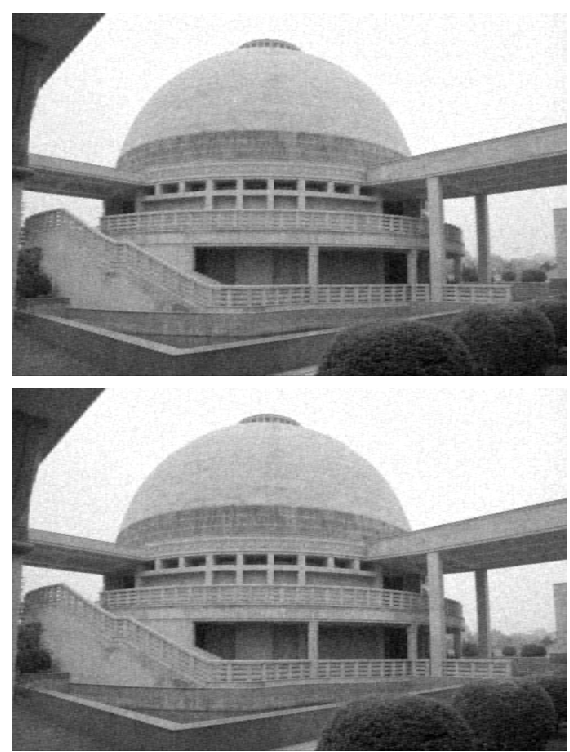

(a)

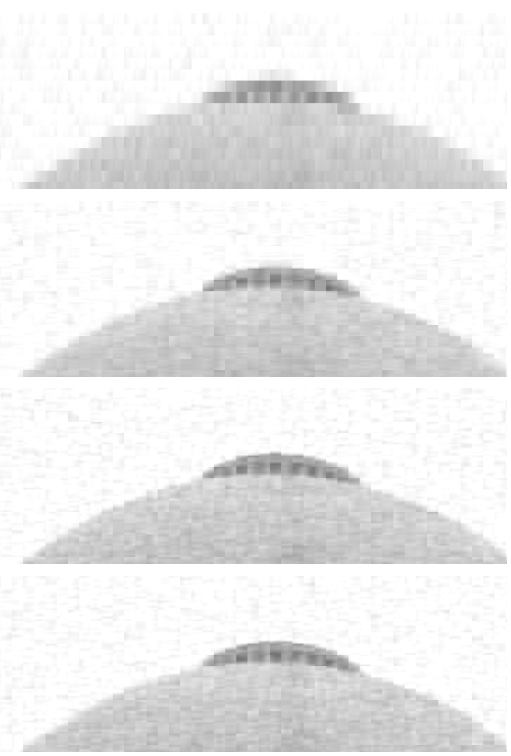

(b)
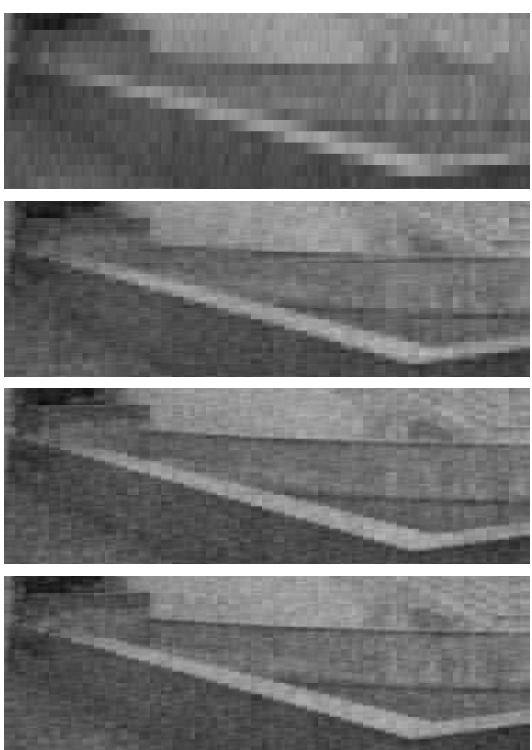

(c)

Fig. 2. The object reconstruction using (a) the optimal binary matrix (top) and PCA (bottom); (b)\&(c) using Hadamard sorted by variation in a vector, by projections of $\mathbf{x}_{\text {mean }}$ over Hadamard, the optimal, and PCA (from top to bottom). Noise level is $\sigma$ is 150 .

\section{Conclusion}

In this work, a binary sensing matrix is searched for CI, by solving an optimization problem which is to minimize the difference between the matrix and PCA. Using a numerical experiment, we find the solution to the optimization problem presents the best reconstruction among binary matrices and comparable reconstruction quality as PCA.

This work was supported in part by the National Natural Science Foundation of China under Project 61307022, and by the Research Grants Council of the Hong Kong Special Administrative Region, China, under Projects HKU $7138 / 11 \mathrm{E}$ and 7131/12E.

\section{References}

1. Michael Elad. Optimized projections for compressed sensing. IEEE Transactions on Signal Processing, 55(12):5695-5702, December 2007.

2. Duarte-Carvajalino, Julio Martin, and Guillermo Sapiro. Learning to sense sparse signals: Simultaneous sensing matrix and sparsifying dictionary optimization. IEEE Transactions on Image Processing, 18(7):1395-1408, July 2009.

3. Wei Chen, Miguel R.D. Rodrigues, and Ian J. Wassell. Projection design for statistical compressive sensing: A tight frame based approach. IEEE Transactions on signal processing, 61(8):2016-2029, April 2013.

4. Jun Ke, Michael D. Stenner, and Mark A. Neifeld. Minimum reconstruction error in feature-specific imaging. In Proceedings of SPIE, volume 5817, pages 7-12. SPIE, 2005.

5. R. Baldick. Applied Optimization: Formulation and Algorithms for Engineering Systems. Cambridge University Press, first edition, 2006.

6. Mark A. Neifeld and Jun Ke. Optical architectures for compressive imaging. Applied Optics, 46(22):52935303, July 2007.

7. Jun Ke and Edmund Y. Lam. Object reconstruction in block-based compressive imaging. Optics Express, 20(20):22102-22117, September 2012.

8. Jun Ke, Ping Wei, Xin Zhang, and Edmund Y. Lam. Block-based compressive low-light-level imaging. In IEEE International Conference on Imaging Systems and Techneques. IEEE, 2013. 